\title{
Formation and disappearance of a filament
}

\author{
G. P. Zhou ${ }^{1}$, J. X. Wang ${ }^{1}$ and S. Tsuneta ${ }^{2}$ \\ ${ }^{1}$ National Astronomical Observatories, Chinese Academy of Science, Beijing, China 100012 \\ email: gpzhou@nao.cas.cn \\ ${ }^{2}$ National Astronomical Observatory of Japan, Mitaka, Tokyo \\ email: saku.tsuneta@nao.ac.jp
}

\begin{abstract}
A continuous observations near an small active region (AR) NOAA 10976 by the Solar Optical Telescope (SOT) and X-Ray Telescope (XRT) on board the Hinode satellite during Dec. 022007 from 15:50 UT to 19:59 UT. We observed that: (a) the filament formed by merging parallel fibrils into a twist structure. (b) the filament faded by reconnecting among its own loop structures, or being stripped into pieces. These process are always preceded by the brightening. (c) for the disappearance of the filament, it has close correlation with the activity, like untwisting or expanding, of its corresponding X-ray bright rope.
\end{abstract}

Keywords. Filament, Flux rope.

\section{Observations \& Discussions}

The predominant data sources in this work are $\mathrm{H} \alpha$ images from Solar Optical Telescope (SOT), soft X-ray images from X-Ray Telescope (XRT), the vector images from the Stokes Polarimeter (SP) on Hinode. The filaments are observed by Hinode SOT/Narrowband Filter Imager (NFI) at the wavelength of H I $6563 \AA$, with the wavelength offset from the line center is $-132 \mathrm{~m} \AA$, and a typical temporal cadence of about $20 \mathrm{~s}$ and a pixel size of 0.16. All the sequenced data are compensated for the solar rotation and are aligned accurately. The XRT images are aligned with SOT magnetograms according to Shimizu et al. (2007).

Based on the series of Halpha observation with high temporal and spatial resolutions, we can observe the formation, fading away, and disappearance of a filament. For the formation of a filament, we exactly identified the mergence of parallel fibrils into a twist structure. For the fading of a filament, there are three modes: (a) reconnection among filaments own loop structures; (b) being stripped into pieces; (c) being broken into parallel fibres. This process is always preceded by the brightening which may indicate a reconnection. For the disappearance of the filament, it is affected by its corresponding flux rope. Accompanying loosing or expanding of the corresponding XRT bright loops, the filament structure fragmented and disappeared in the end.

\section{Acknowledgements}

The work is supported by the National Natural Science Foundation of China (10973019, 11003024), and the National Key Basic Research Science Foundation (G2011CB811403 and G2011CB811402). 


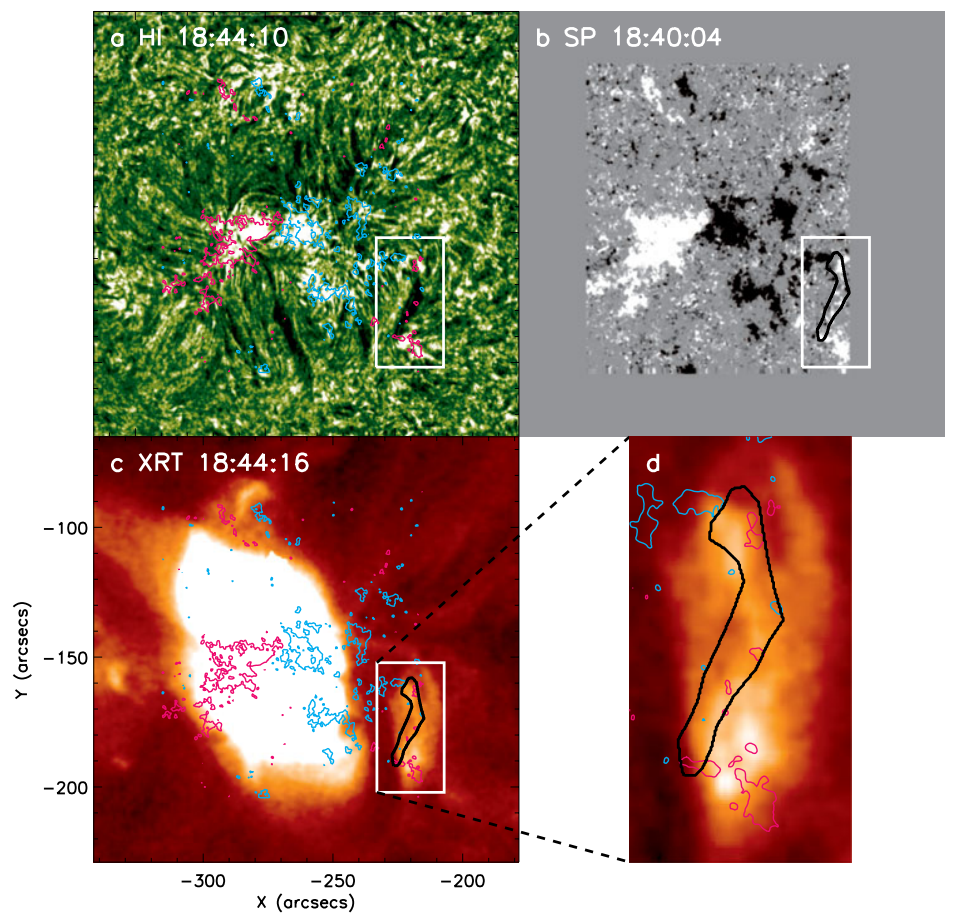

Figure 1. Hinode observations at different wavelengths of HI (a), SP (b), and XRT (c) are shown in the same field of view. The pink and blue contours indicate the SP data of \pm 1000 G. The black contours indicate the filament. Panel (d) is a enlarged XRT image in the white quadrate regions.

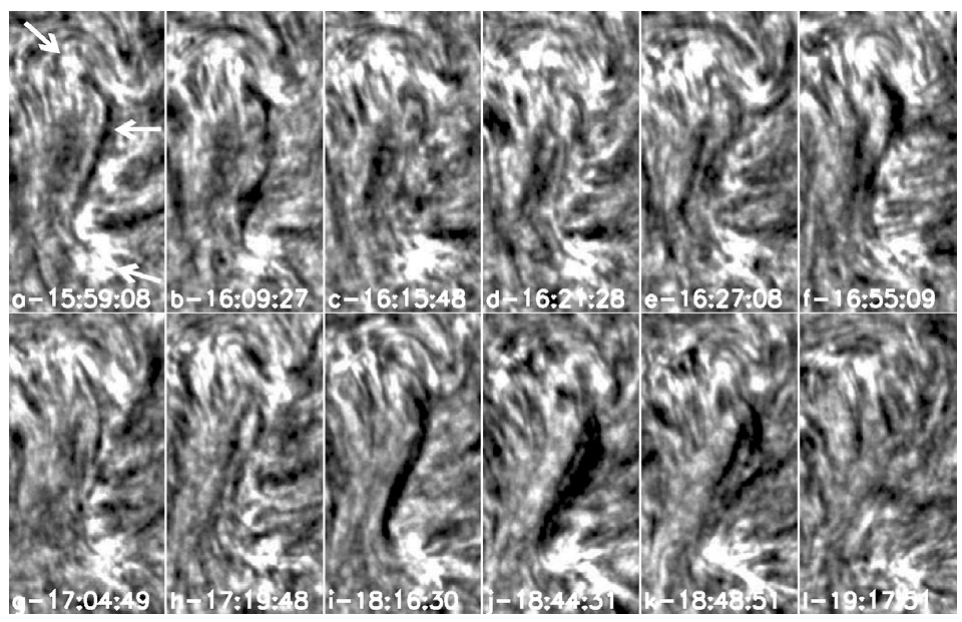

Figure 2. $\mathrm{H} \alpha$ observations of a filament (see middle arrow in a). It is located between two bright footpoints (see upper and lower arrows in a). It faded by reconnecting among its own loops (b-c), formed flux rope by merging paralleled fibres (d-f, h-i), faded by being stripped into pieces (f-g), and disappeared with corresponding X-ray helical loops untwisting or expanding $(\mathrm{i}-\mathrm{l})$.

\section{Reference}

Shimizu, T., Katsukawa, Y., Matsuzaki, K. et al. 2007, PASJ, 59, 845 